\title{
Start ins dritte Jahr der Kampagne: Das Medizinal- und Pflegepersonal im Fokus
}

\author{
Nationale Grippepräventionskampagne des Bundesamtes für Gesundheit 2001 - 2005
}

\author{
D. A. Müller
}

1 Potter J, Stott DJ, Roberts MA, Elder AG, O'Donnell B, Knight PV, Carman WF. Influenza vaccination of health care workers in longterm-care hospitals reduces the mortality of elderly patients. J Inf Dis 1997; 175:1-6.

2 Carman WF, Elder AG, Wallace LA, McAulay K, Walker A, Murray GD, Stott DJ. Effects of influenza vaccination of health-care workers on mortality of elderly people in long-term care: a randomised controlled trial. Lancet 2000; 355:93-7.

Korrespondenz:

Daniela A. Müller, dipl. biol. Bundesamt für Gesundheit Abteilung Epidemiologie und Infektionskrankheiten Projektleitung Grippeprävention Postfach CH-3003 Bern

\section{Einleitung}

In Spitälern, in der Arztpraxis, in Alters- und Pflegeheimen sowie überall dort, wo das Medizinal- und Pflegepersonal in nahem Kontakt steht zu Personen mit einem erhöhten Komplikationsrisiko infolge einer Grippe, birgt die Übertragung von Influenzaviren eine erhöhte Gefahr. Indem sich das Medizinal- und Pflegepersonal gegen Grippe impfen lässt, schützt es sich selbst vor einer Erkrankung, und gleichzeitig wird das Risiko der Übertragung auf Patientinnen und Patienten reduziert. Wissenschaftliche Studien zeigen, dass Grippeerkrankungen und Todesfälle bei Bewohnerinnen und Bewohnern von Pflegeinstitutionen markant reduziert werden können, wenn sich die im Medizinal- und Pflegebereich tätigen Personen gegen Grippe impfen lassen $[1,2]$.

Die vom Bundesamt für Gesundheit im Jahr 2001 mit Unterstützung der FMH lancierte Nationale Grippepräventionskampagne steht vor dem Start ins dritte Jahr. Ziel der mehrjährigen Kommunikations- und Informationskampagne ist die Förderung der Grippeprävention bei Personen mit einem erhöhten Komplikationsrisiko nach einer Influenzaerkrankung sowie beim Personal im Gesundheitswesen. Im Jahr 2003 richtet sich die Kampagne in erster Linie an das Medizinal- und Pflegepersonal, das in verschiedenen Bereichen mit der Betreuung, Behandlung und Pflege von Patientinnen und Patienten betraut ist.

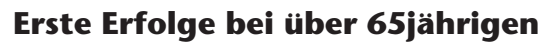

Während sich die Kampagne im Jahr der Lancierung mit Schwergewicht an die Ärzteschaft und an Apothekerinnen und Apotheker gerichtet hat, wurden im Jahr 2002 in erster Linie über 65jährige Personen angesprochen. Die Evaluation der Kampagne bei dieser Zielgruppe, die in der Schweiz etwa 1 Mio. Personen umfasst, führte zu wertvollen Erkenntnissen, die auch in Zukunft zur Förderung der Grippeprävention von Bedeutung sein werden. Erfreulicherweise lassen sich in der Schweiz Jahr für Jahr mehr ältere Menschen gegen Grippe impfen. Seit 1999 kann in dieser Bevölkerungsgruppe eine kontinuierliche Zunahme der Durchimpfung festgestellt werden. Im Herbst 2002 haben sich aufgrund einer Telefonumfrage $(n=2347)$ rund $55 \%$ der über 65jährigen gegen Grippe impfen lassen. Knapp zwei Drittel der Befragten (65\%), die im Herbst 2002 mindestens einmal eine Ärztin / einen Arzt konsultierten, erinnern sich, dass die Grippeimpfung von der Ärztin/vom Arzt thematisiert wurde. Nach dem Motivationsgrund für eine Impfung befragt, wird an erster Stelle die Ärztin / der Arzt erwähnt. So lassen sich rund ein Drittel der Befragten (34\%) auf Anraten der betreuenden Ärztin / des Arztes impfen. An zweiter Stelle (21\%) werden Präventionsgründe als Motivation für eine Impfung angegeben.

Diese Ergebnisse machen die wichtige Schlüsselstellung der Ärzteschaft im Bereich der Förderung der Grippeprävention bei älteren Personen deutlich. Ärztinnen/Ärzte sind für ältere Personen und auch für Risikopersonen mit einem erhöhten Komplikationsrisiko die wichtigsten Ansprechpartner.

Es wird jedoch durch diese Evaluationsstudie auch deutlich, dass zur Förderung der Grippedurchimpfung bei über 65jährigen weitere Informations- und Sensibilisierungsarbeit nötig ist. Diejenigen, die sich nicht gegen Grippe impfen lassen, sind in erster Linie der Meinung, es sei nicht nötig (19\%). Als zweiter Grund gegen eine Grippeimpfung bei Nicht-Geimpften wird die negative Einstellung zu Impfungen generell (15\%) erwähnt.

Rund die Hälfte der Studienteilnehmer (52\%) hat die Grippepräventionskampagne des BAG bewusst wahrgenommen. Die primären Informationsquellen sind Artikel in der Tagesund Wochenpresse, Inserate in verschiedenen Zeitschriften und die Broschüren des BAG. 


\section{Durchimpfung beim Personal im Gesundheitsbereich}

Aufgrund einer im März 2003 in der Schweiz durchgeführten Umfrage bei Ärztinnen und Ärzten mit Fachspezialisierung in Allgemeinmedizin und Innerer Medizin sowie bei Personen mit einer Ausbildung im Medizinal- und Pflegebereich $(n=4276)$ hat sich im Herbst 2002 gesamtschweizerisch knapp ein Drittel (28,3 \%) des Personals im Gesundheitsbereich mit direktem Kontakt zu Patientinnen und Patienten gegen Grippe impfen lassen. Beim Medizinal- und Pflegepersonal liegt die Durchimpfung bei $21 \%$, während der Anteil bei der Ärzteschaft rund drei Viertel (74,6 \%) beträgt. Die Durchimpfung nach Sprachregionen präsentiert sich sehr unterschiedlich. Beim Medizinal- und Pflegepersonal (ohne Ärzteschaft) ist die Durchimpfung in der Romandie $(27,8 \%)$ signifikant höher als in der

\section{Die Kampagne}

Die Kampagne richtet sich an Personen über 65 Jahre, an Personen mit chronischen Erkrankungen, an Bewohnerinnen und Bewohner von Alters- und Pflegeheimen, an Personen, die regelmässig medizinische Betreuung benötigen sowie an das Medizinal- und Pflegepersonal [3]. Durch gezielte Information wird, basierend auf wissenschaftlichen Erkenntnissen, auf die Nutzen der Grippeimpfung und auf die möglichen Gefahren einer Grippeerkrankung, insbesondere bei Risikopersonen, aufmerksam gemacht.

Informieren, Sensibilisieren und Erklären sind die Schwerpunkte dieser Kampagne. Gezielt informiert wird einerseits das Fachpersonal im Gesundheitsbereich (Ärzteschaft, Apothekerinnen / Apotheker, Spitalverwaltungen, Spitex-Mitarbeitende usw.), welches bei der Förderung der Durchimpfung eine wichtige Rolle einnimmt, und andererseits Risikopersonen oder Personen, die in nahem Kontakt stehen zu Risikopersonen. Ziel der mehrjährigen Kampagne ist die Erhöhung der Durchimpfung auf $60 \%$ bei über 65 jährigen und auf $70 \%$ bei den übrigen Risikogruppen sowie beim Medizinal- und Pflegepersonal. Das primäre Zielpublikum des Jahres 2004 werden Personen mit chronischen Erkrankungen sowie Bewohnerinnen und Bewohner von Altersund Pflegeheimen sein. deutschen Schweiz (19,7 \%), während sie im Vergleich zum Tessin (24,6\%) nicht bedeutend höher ist. Bei Ärztinnen und Ärzten ist die Durchimpfung signifikant höher in der Romandie (80,2\%) als im Tessin (62,7\%); es zeigt sich jedoch kein grosser Unterschied zur Durchimpfung der Deutschschweizer Ärzteschaft (73,6 \%).

Sehr unterschiedlich präsentiert sich die Durchimpfung in verschiedenen Arbeitsbereichen wie Spitälern, Alters- und Pflegeheimen, bei der Spitex oder in Arztpraxen. In den Altersund Pflegeheimen lassen sich aufgrund der Erhebung unter dem Personal (ohne Ärzteschaft) signifikant mehr Personen impfen (26\%) als in Spitälern (17\%) und bei der Spitex (16\%). Am höchsten ist die Durchimpfung des Personals in der Arztpraxis (39\%). Bei Ärztinnen und Ärzten hingegen ist die Durchimpfung höher bei Spitalangestellten $(83,2 \%)$ als bei Personen mit Praxistätigkeit $(74,4 \%)$.

In bezug auf die Einstellung zur Grippepräventionskampagne des BAG äussert ein Grossteil der Ärztinnen und Ärzte (83,8 \%) eine positive Haltung. Beim Medizinal- und Pflegepersonal sind der Kampagne gegenüber ungefähr gleiche Anteile positiv eingestellt (40\%) oder vertreten diesbezüglich keine klare Meinung $(39,5 \%)$.

An dieser Stelle möchten wir die Gelegenheit wahrnehmen und uns bei all denjenigen, die an der Umfrage teilgenommen und den Fragebogen zurückgesandt haben, bestens bedanken.

\section{Inhalt der Kampagne 2003}

Im dritten Jahr der Grippepräventionskampagne, die in enger Zusammenarbeit mit der Kantonsärzteschaft erfolgt, steht das Medizinalund Pflegepersonal im Zentrum der Kommunikationstätigkeit des BAG. Die Erfahrungen und Erkenntnisse aus dem vergangenen Kampagnejahr, die in Kollaboration mit dem Spitex-Dachverband durch die Spitex-Mitarbeitenden gewonnen werden konnten, werden für die diesjährigen Tätigkeiten miteinbezogen.

Neu gibt es dieses Jahr eine Broschüre, die sich spezifisch an das Medizinal- und Pflegepersonal richtet. Sie liefert, basierend auf dem neusten Stand der Wissenschaft, Fakten zur Grippe und Grippeimpfung speziell im Pflegebereich, aber auch ganz generell. Weiter steht dieses Jahr neu ein Informationskit mit einer Auswahl an Hilfsmitteln zur Verfügung. Das Kit soll denjenigen Personen im Medizinal- und Pflegebereich, die für die Organisation und Durchführung von Weiterbildungs- und Infor- 
mationsveranstaltungen zuständig sind, die Arbeit erleichtern. Eine Checkliste liefert beispielsweise Ideen, wie in verschiedenen Institutionen auf unterschiedliche Art und Weise Impfaktionen durchgeführt werden können, wie das Personal für eine Impfung motiviert werden kann oder auch mit welchen Widerständen gerechnet werden muss. Eine PowerPoint-Präsentation mit Notizen auf CD dient den Informations- und Weiterbildungszwecken. Das Kit beinhaltet weiter farbige Poster im Format A2, die das Sujet der Kampagne 2003 zeigen und zusätzlich für Ergänzungen per Handschrift Platz bieten, so dass die Poster je nach Bedarf, z. B. zur Ankündigung einer Impfveranstaltung, individuell eingesetzt werden können. Die in den ersten beiden Jahren der Kampagne eingeführten Informationsmittel, die Broschüre für Fachpersonen im Gesundheitswesen sowie die Broschüre mit Kurzinformationen für Patientinnen und Patienten, werden im Jahr 2003 überarbeitet und neu gestaltet.

Die Informationsmittel werden der Ärzteschaft vor Beginn der Impfperiode kostenlos zur Verfügung gestellt. Ärztinnen / Ärzte, Apothekerinnen / Apotheker, Zahnärztinnen / Zahnärzte und alle Berufs- und Fachverbände werden dieses Jahr je ein Belegexemplar der neuen Broschüre «Vielen Dank!» für das Medizinal- und Pflegepersonal, der Broschüre für Fachpersonen im Gesundheitswesen sowie der Broschüre mit Kurzinformationen für Patientinnen und Patienten erhalten. Ein zusätzlicher Bestelltalon bietet die Möglichkeit für individuelle Bestellungen sämtlicher Informationsmittel und Poster je nach Bedarf. Ein Infokit mit einer Checkliste, einer CD mit Vortrag, mehreren Exemplaren der drei verschiedenen Broschüren und mit Poster wird an Spitäler und Kliniken, an Alters- und Pflegeheime, an Spitex-Organisationen und an Ausbildungszentren für Pflegepersonal versandt.

Die Internetseite wird laufend aktualisiert und bietet die Möglichkeit, die verschiedenen Dokumente kostenlos zu bestellen. Weitergeführt wird zudem die Zusammenarbeit mit Medgate, dem medizinischen Beratungszentrum, das für die breite Bevölkerung eine Impfline mit
Informationen zu Grippe- und Routineimpfungen anbietet. Inserate in Zeitschriften wie CoopZeitung und Brückenbauer weisen zwischen Mitte Oktober und Mitte November auf die Grippeimpfung resp. auf den Zeitpunkt für die Impfung hin. Ferner werden am Radio während der Impfperiode in der Romandie die «Meteo»Sendungen und die Verkehrsvorhersage und im Tessin die «Meteo»-Sendungen von einem Aufruf zur Grippeimpfung an Seniorinnen und Senioren begleitet.

\section{Informationsquellen}

Auf der Internetseite der Grippeprävention, unter www.grippe.admin.ch, erhalten Sie weitere Auskunft über die nationale Grippepräventionskampagne des Bundesamtes für Gesundheit. Sämtliche Informationsunterlagen können in drei Landessprachen kostenlos bei der Dokumentationsstelle Grippeprävention telefonisch (031 35214 60), per Fax (031 35214 71) oder per E-Mail (grippe@bag.admin.ch) bestellt werden. Die Empfehlungen zur Grippeprävention (Supplementum XIII, in Deutsch, Französisch und Italienisch) sind im Internet $\mathrm{zu}$ finden unter www.bag.admin.ch/infekt/impfung/vaccs/d/in dex.htm oder können unter folgender Adresse bezogen werden: BAG, Sekretariat, Abteilung Epidemiologie und Infektionskrankheiten, 3003 Bern, Telefon 03132387 06, Fax 0313238795. Die Medgate Impfline des medizinischen Beratungszentrums Medgate ist unter der Nummer 0844448448 erreichbar.

Informationen zur Grippesituation in der Schweiz und in Europa finden Sie im Internet unter www.bag.admin.ch/sentinella (Homepage der Sentinella-Meldesysteme, BAG), unter www.influenza.ch (Homepage des Nationalen Zentrums für Influenza in Genf) und unter www.eiss.org (European Influenza Surveillance Scheme [EISS]). Weitere Informationen zur Grippe im allgemeinen, zu ihrer Verhütung und Behandlung liefert die Arbeitsgruppe «Unis contre la grippe» auf www.grippe.ch. 